\title{
Lightning induced atrial fibrillation
}

\section{Dronacharya $\mathbf{L}^{1}$, Poudel $\mathbf{R}^{2}$}

${ }^{1}$ Intern, Manipal Teaching Hospital, Pokhara, ${ }^{2}$ Lecturer, Department of Medicine, Manipal Teaching Hospital, Pokhara, Nepal

\begin{abstract}
Atrial fibrillation (AF) is a common arrhythmia that occurs in paroxysmal and persistent forms. It occurs in varied situations but lightning induced AF is extremely rare. Here is a case which reverted to sinus rhythm spontaneously. This 37-year-old man without any underlying heart disease had new onset AF after being struck by a lightning. Oral Metoprolol alone was given to control ventricular rate. Spontaneous reversion to sinus rhythm within 36 hours is in favor of new onset lightning induced AF.
\end{abstract}

Key words: Lightning injuries, atrial fibrillation, ECG changes

$\mathbf{L}$ ightning produces manifold systemic effects of varying severity. Notable amongst them are neurological and cardiovascular ones ${ }^{1}$. Transient and persistent atrial fibrillation, which has its many causes, can result from lightning mainly due to its effect on autonomic nervous system. Due to its hemodynamic effects and other serious cardiovascular injuries, patients struck by lightning should be evaluated completely to detect these effects. Patient with cardiac arrhythmia should be admitted and monitored by continuous Electrocardiography (ECG). Here, we report a case of lightning induced AF which reverted spontaneously to sinus rhythm.

\section{Case report}

The patient, 37 year old man, was struck by a lightning when he was standing in his front yard during rain. He was unresponsive, according to bystanders, for almost five minutes after which he regained full consciousness. He did not receive any kind of resuscitation on the spot. He had vomited five times since then. When he presented

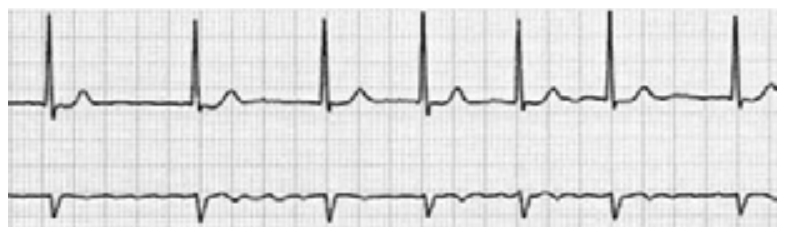

Fig 1: ECG tracing shows irregular ventricular rhythm associated with poorly defined atrial activity consistent with atrial fibrillation to emergency department after three hours, he was fully conscious and stable. However he is a deaf and dumb person since birth. There was no history of any kind of cardiorespiratory disease. Vital signs taken at that time revealed pulse rate of 116 beats per minute, irregularly irregular with apex pulse deficit of 19 per minute, blood pressure $150 / 80 \mathrm{mmHg}$, respiratory rate $18 /$ min and temperature 98.6 $6^{\circ}$ Fahrenheit. General survey of the whole body did not show any physical injuries and fixed entry and exit points. Systemic examination was unremarkable. Electrocardiogram taken at that time confirmed the diagnosis of atrial fibrillation (Figure 1). All the laboratory investigations including creatine phosphokinase (CPK) and cTroponin I (cTnI) were negative. There was no laboratory evidence of myoglobinuria. He was then admitted to Intensive Care Unit (ICU) for next 36 hours with continuous ECG monitoring. He was given oral Metoprolol $25 \mathrm{mg}$ BID and Aspirin $75 \mathrm{mg}$ OD. After 36 hours it reverted to sinus rhythm and was discharged without any medication (Fig 2).

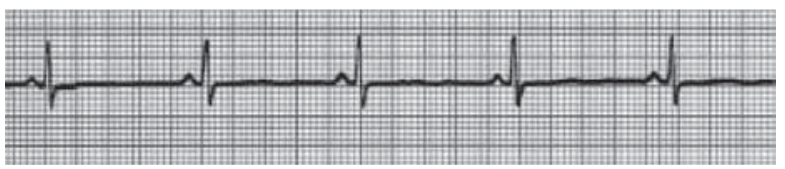

Fig 2: Normal ventricular rhythm after 36 hours

\section{Correspondence}

Dr. Dronacharya Lamichhane

Manipal College of Medical Sciences/MTH

Deep Height, Pokhara, Nepal

E-mail: dreamysu@hotmail.com 


\section{Discussion}

Lightning produces a massive unidirectional current which produces multisystem injuries of varied severity ${ }^{1}$. Over the last century, records of environmental injuries and mortality indicate that lightning has consistently been one of the top three environment related cause of death. Lightning fatality rate is $8-10 \%$. Cardiac arrest at the time of injury is usually the cause of death. Lightning causes injury through five basic mechanisms: direct strike, flash discharge (splash), contact, ground current (step voltage), and blunt trauma ${ }^{2}$.

Different types of cardiovascular injuries have been reported so $\mathrm{far}^{3}$. These include primary and secondary cardiac arrest, myocardial injury (infarction and ischemia), arrhythmias and other ECG abnormalities. ECG changes may not occur until late in the course and hence it is a poor diagnostic tool. Many changes have been reported but the most common is QT prolongation. But so far, atrial fibrillation has been reported only twice $^{4,5}$. Commonly accepted dogma is that the heart after standstill, restarts in sinus bradycardia, but is overcome once more by a secondary hypoxic arrest due to pulmonary standstill.

Andrews et al. have reported experiments suggesting the sequence to be asystole followed by a small period of bradycardia, the intense tachycardia leading to secondary hypoxic arrest ${ }^{2}$. Ventricular sinus pauses may be seen as many sinus pauses with escape. Ventricular fibrillation has been recently reported. Ventricular tachycardia during treatment has been seen. It is unknown whether cardiac arrest and arrhythmias induced by lightning are a result of damage to central cardiac or respiratory centers in the brain, to the carotid body and other pacemakers along with other carotid control path, to feedback control mechanisms within the autonomic nervous centre, or to a combination of these. Certainly, clinical evidence of general damage to autonomic nervous system regulation has been well documented. Recently, cardiac effects from autonomic nervous system damage have been confirmed in animal laboratory ${ }^{2}$. Management of lightning induced cardiac dysrhythmias is not different from that of other etiologies. Because persons struck by lightning have a better chance of survival than persons who experience cardiopulmonary arrest from other causes, resuscitation for persons struck by lightning must be instituted immediately, followed by a comprehensive treatment program of the other systemic manifestations. Fortunately, electrocardiographic changes usually resolve without serious cardiac complications. Prompt symptomatic improvement has occurred after the intravenous administration of diuretics. Obviously, the victim of lightning who is severely injured would need admission, often to an intensive care unit. Especially if a patient has evidence of cardiac dysrhythmias or myocardial injury or has been revived from cardiorespiratory arrest, he or she should be admitted to an appropriate monitored setting including continuous ECG.

The previously reported two cases of atrial fibrillation had reverted to sinus rhythm spontaneously ${ }^{4,5}$. However in acute atrial fibrillation with hemodynamic compromise, electrical cardioversion is the treatment of choice. In the absence of severe cardiovascular compromise, slowing of the ventricular rate becomes the initial therapeutic goal. This may be most rapidly accomplished with beta adrenergic blockers and or calcium channel antagonists.

\section{Conclusion}

Cardiac injury is the major cause of morbidity and mortality among the lightning related injuries. Cardiopulmonary arrest is the usual cause of death. Myocardial infarction/ischemia, arrhythmia, and ST-T changes have been reported. However it can be one of the causes of acute atrial fibrillation. The management aspect of it does not significantly differ from that of other etiologies.

\section{References}

1. Cooper MA, Andrews CJ, Holle RL, Lopez RE. Lightning Injuries. In: Auerbach P, editor. Wilderness Medicine, $4^{\text {th }}$ edition. St Luis: Mosby; 2000. p 77-111.

2. Andrews CJ, Dauveniza M, Mackeras D. Lightning Injuries A Review of clinical Aspect, Pathophysiology and Treatment. Advances in Trauma 1989;4:241-87.

3. Lichtenberg R, Dries D, Ward K, Marshall W, Scanlon P. Cardiovascular effects of lightning strikes. J Am Coll Cardiology 1993(Feb);21(2):531-6.

4. MorganZV, Headley RN, Alexander EA, Sawyer CG. Atrial Fibrillation and Epidural Haematoma associated with Lightning Stroke. New Eng. J. Med. 1958;259(20):956-9.

5. Gupta GB, Gupta SR, Somani PN, Agrawal BV. Atrial fibrillation with inferior wall myocardial ischemia following lightning. J Assoc Physician India. 1988( May);36(5):354-5. 